\title{
Effect of resveratrol on endocrine function, oocyte oxidation and apoptosis in mice with polycystic ovary syndrome
}

\author{
Weiyan Huang ${ }^{1}$, Lili Duan², Meiyu $\mathbf{W u}^{3}$, Lei Wang ${ }^{4}$, Zhifang Wang ${ }^{5 *}$ \\ ${ }^{1}$ Department of Gynecology, Zhuji People's Hospital, Zhuji 311800, Zhejiang Province, ${ }^{2}$ Department of Obstetrics, The Eighth \\ People's Hospital of Hengshui, Hengshui 253800, Hebei Province, ${ }^{3}$ Department of Gynecology, Shangrao Municipal Hospital, \\ Shangrao 334000, Jiangxi Province, ${ }^{4}$ Department of Pharmacy, Deyang People's Hospital, Deyang 618000, Sichuan Province, \\ ${ }^{5}$ Department of Gynaecology, 521 Hospital of Norinco Group, Xi'an 710000, Shaanxi Province, China
}

*For correspondence: Email: jrsrf1@163.com

Sent for review: 19 January 2021

Revised accepted: 27 April 2021

\begin{abstract}
Purpose: To investigate the effect of resveratrol on endocrine function, oocyte oxidative stress and oocyte apoptosis in mice with polycystic ovary syndrome (PCOS).

Methods: Healthy female Kunming mice $(n=180)$ were assigned to control, model and resveratrol groups, respectively. Changes in ovarian tissue in each group, including apoptosis of granulosa cells, were measured. Oocyte abnormal mitochondria, changes in oocyte mitochondrial membrane potential, expression levels of $\mathrm{Bcl}-2$, Bax and caspase-9 in oocytes evaluated. The activities of glutathione peroxidase (GPX) and superoxide dismutase (SOD), and levels of reactive oxygen species (ROS) in each group were also assayed.

Results: Compared with the model group, the levels of sex hormones, ovarian cell apoptosis and abnormal mitochondria of mice in the resveratrol group were significantly reduced, while uterine weight increased ( $p$ <.05). The expression levels of Bax, Bcl-2, caspase-9, GPX and SOD, and ROS levels in the resveratrol group decreased significantly $(p<0.05)$.

Conclusion: Resveratrol significantly improves endocrine function in PCOS mice, but inhibits oocyte apoptosis and oxidative stress response. Thus, resveratrol may be useful in the treatment of PCOS but further clinical trials are required to confirm it.
\end{abstract}

Keywords: Resveratrol, Polycystic ovary syndrome, Endocrine function, Oocyte oxidative stress, Apoptosis

\begin{abstract}
This is an Open Access article that uses a fund-ing model which does not charge readers or their institutions for access and distributed under the terms of the Creative Commons Attribution License (http://creativecommons.org/licenses/by/4.0) and the Budapest Open Access Initiative (http://www.budapestopenaccessinitiative.org/read), which permit unrestricted use, distribution, and reproduction in any medium, provided the original work is properly credited.

Tropical Journal of Pharmaceutical Research is indexed by Science Citation Index (SciSearch), Scopus, International Pharmaceutical Abstract, Chemical Abstracts, Embase, Index Copernicus, EBSCO, African Index Medicus, JournalSeek, Journal Citation Reports/Science Edition, Directory of Open Access Journals (DOAJ), African Journal Online, Bioline International, Open-J-Gate and Pharmacy Abstracts
\end{abstract}

\section{INTRODUCTION}

Polycystic ovary syndrome (PCOS) is an endocrine and metabolic disorder which causes female infertility. According to relevant statistics, the incidence of PCOS in women of child-bearing age is about $13 \%$ [1]. The pathogenesis of PCOS is not yet well understood. However, it is a relatively complex syndrome involving multiple systems, and it may be closely related to insulin resistance and hyperinsulinemia [2]. 
At present, western medical treatment of PCOS in clinics involves the use of insulin sensitizers and oral contraceptives. However, these treatments are associated with adverse side effects such as ovarian hyperstimulation syndrome and lactic acidosis. Therefore, it is important to find a treatment method for PCOS with less adverse reactions. Due to their better therapeutic effects and low adverse reactions, Traditional Chinese Medicines (TCMs) have continued to attract the attention of researchers. In TCM, it is believed that the development of PCOS is linked to kidney deficiency, phlegm turbidity and blood stasis, and that its main treatment strategy should involve tonifying the kidney and resolving phlegm [3].

Resveratrol, a natural plant antitoxin which exists in a variety of natural foods, has anti-bacterial, anti-oxidant and anti-cancer properties. Besides, resveratrol inhibits the expression of vascular endothelial growth factor (VEGF) [4]. Studies have shown that resveratrol mitigates decline in ovarian function [5]. However, the effect of resveratrol on PCOS remains unclear. The present study was carried out to investigate the influence of resveratrol on ovarian tissue and oocytes of mice with PCOS.

\section{EXPERIMENTAL}

\section{Materials}

A total of 180 healthy female Kunming mice (mean body weight $=25 \pm 4 \mathrm{~g}$ ) were obtained from Beijing Vital River Experimental Animal Technology Co. Ltd \{production license no. SCXK (Beijing) 2019-0009; Usage License number: SYXK (Beijing) 2017-0033\}. The animals were permitted ad libitum access to feed and drinking water in an environment with $12 \mathrm{~h}$ light/12 h dark cycle, mean temperature of $23 \pm 2$ ${ }^{\circ} \mathrm{C}$, and relative humidity of $55 \pm 15 \%$.

\section{Main instruments and reagents}

The major instruments and reagents used, and their suppliers (in parenthesis) were: lowtemperature high-speed centrifuge (Hunan Xiangxin Instrument and Meter Co. Ltd, model TGL-18M); electronic balance (Sartoriu Scientific Instruments Co. Ltd, model BSA224s-CW); Paraffin slicer (Shenyang Hengsong Technology Co. Ltd., Model: HS-S7220); $-80^{\circ} \mathrm{C}$ ultra-low temperature refrigerator (Jinan Zhuolong Biotechnology Co. Ltd., Model: BDF-60V50); Biological microscope (Shanghai Yuanren Testing Equipment Co. Ltd, model YRS-32); TUNEL detection kit (Beijing Biolab Technology Co. Ltd); dehydroepiandrosterone (MRM, Inc., specification: 90s); and resveratrol (Shaanxi Diankai Biotechnology Co. Ltd; purity: over 98 $\%)$.

\section{Treatments}

All mice were fed adaptively for 1 week, after which they were assigned to control, model and resveratrol groups, with 60 animals/group.

\section{Establishment of PCOS mouse model}

Dehydroepiandrosterone (DHEA) was dissolved in oil medium to yield a concentration of 6 $\mathrm{mg} / 100 \mathrm{~g}$ oil. The solution was injected into mice subcutaneously every day continuously for 28 days. The occurrence of keratinization in mouse vaginal epithelial cells consecutively for one week was considered as indication that the PCOS model was successfully established. Mice in the control group were injected subcutaneously with oil medium only. Control and model mice were intragastrically administered normal saline, while mice in the resveratrol group were intragastrically administered resveratrol at a dose of $100 \mathrm{mg} / \mathrm{kg}$ body weight. This research was approved by the Animal Ethical Committee of 521 Hospital of Norinco Group (approval no. 20180679) and performed according to the guidelines of "Principles of Laboratory Animal Care" (NIH publication no. 85-23, revised 1985) [6].

\section{Assay of hormonal level}

At the end of treatment, drinking water was removed from mice in each group, and the animals were fasted overnight. Thereafter, $3 \mathrm{~mL}$ of orbital blood was taken from each mouse. The blood samples were allowed to clot, and the serum samples obtained after centrifugation were used for assay of levels of testosterone (T), $\mathrm{LH}, \mathrm{FSH}$ and leptin, with their respective ELISA kits.

Then, the weights of 30 mice from every group were obtained. The ovaries and uteri of the mice were excised and weighed.

\section{Ovarian histology}

Ovarian tissues were processed for light microscopy, and the resultant $\mathrm{H}$ \& E-stained paraffin sections were examined microscopically and photographed.

\section{Determination of apoptosis}

Apoptosis of granulosa cells in ovarian tissue of mice was determined with TUNEL method. 
Paraffin sections were prepared and incubated with $3 \%$ hydrogen peroxide solution in the dark for $20 \mathrm{~min}$, followed by use of $100 \mathrm{~mL}$ sodium citrate buffer for antigen recovery. The slides were heated in a microwave oven and naturally cooled to laboratory temperature, followed by addition of $50 \mu \mathrm{L}$ of TUNEL reaction mixture to every section. The sections were placed in a wet box and incubated at $37^{\circ} \mathrm{C}$ for $1 \mathrm{~h}$, followed by rinsing with phosphate buffer. Then, DAB chromogenic solution was added, and the reaction was terminated after color development. The sections were re-stained with hematoxylin, dehydrated, cleared and sealed, and observed under a microscope. The result of staining was considered as positive if a yellowish-brown or sepia color was seen under the microscope.

The remaining mice were sacrificed, and their ovaries were removed, cut into smaller sections and immersed in balanced culture medium. Oocytes at the germinal vesicle stage, with full morphology and complete germinal vesicle were selected and cultured in an incubator (30 oocytes from each of the three groups). The oocytes were fixed in fixation solution, centrifuged in a low-speed centrifuge, and the supernatant was removed. Agar blocks were placed, embedded with epoxy resin, and sectioned. The oocytes were double-stained with uranyl acetate and lead citrate, and the abnormal mitochondria in the oocytes were observed via transmission electron microscopy. The oocytes were cultured in mitochondrion-fluorescent probe droplets, and JC-1 working solution was added and shaken to bring the JC-1 fluorescent probe in full contact with the oocytes. Care was taken to ensure that the staining processes were carried out in the dark.

After culturing, the oocytes were rinsed with $\mathrm{JC}-1$ buffer in an ice bath. Thereafter, the oocytes were stained within $30 \mathrm{sec}$, and the ratio of redto-green fluorescence was determined using a laser scanning confocal microscope. This ratio is the value of mitochondrial membrane potential.

Changes in ROS levels in oocytes were measured using DCFH-DA method. The oocytes in the germinal vesicle stage were taken up in DCFH-DA working solution, incubated in the dark for $30 \mathrm{~min}$, washed, and sealed with a fluorescence quenching-resistant sealing agent, photographed and observed under a microscope.

\section{Quantitative real-time polymerase chain reaction (qRT-PCR)}

The mRNA expression levels of Bax, Bcl-2, caspase-9, GPx and SOD in oocytes in each group of mice were determined with real-time quantitative PCR. The oocytes were lysed with lysis buffer. Then, $0.2 \mathrm{~mL}$ of chloroform was added, and the mixture was fully shaken and allowed to stand, after which it was centrifuged and the supernatant was removed. Then, isopropanol was added, and the previous steps were repeated, followed by natural drying. The RNA extract was solubilized in $40 \mu \mathrm{L}$ of deionized water and cryopreserved for further use. Then, $2 \mu \mathrm{g}$ RNA was used to synthesize first strand cDNA using reverse transcription reaction, in line with the procedure indicated in the kit. In the PCR reaction, $20 \mu \mathrm{L}$ of cDNA was thoroughly mixed with $80 \mu \mathrm{L}$ of distilled water to complete the premix of template and primer, respectively. $\beta$-Actin was used as internal reference, and the quantitative determination of relative expression levels was carried out with the $2^{-\triangle \Delta C T}$ method. The primer sequences used are shown in Table 1.

Table 1: Primer sequences used in PCR

\begin{tabular}{llc}
\hline Gene & & Sequence5' 3' \\
\hline Bax & F & GCCATCAATGGCAACCCATC \\
& R & CAGCCACCCTGGTCTTGG \\
Bcl-2 & $F$ & GGTGGGGTCATGTGTGTGG \\
& R & GGTTCAGGTACTCAGTCATCC \\
Caspase-9 & F & CGTGACAAACTTGAGCACCG \\
& R & TCTCCATCAAAGCCGTGACC \\
GSH-Px & F & CCTCAAGTACGTCCGACCTG \\
& R & CAATGTCGTTGCGGCACACC \\
SOD & F & AAAGCGGTGTCGTGCTGAA \\
& R & CAGGTCTCCAACATGCCTCT \\
\hline
\end{tabular}

\section{Western blotting}

The protein concentrations of caspase-9, Bax, $\mathrm{Bcl}-2, \mathrm{GPx}$ and SOD were determined with western blotting assay. The oocytes were lysed and centrifuged, and the supernatant was stored in an ultra-low temperature refrigerator at $-80^{\circ} \mathrm{C}$. The protein content of each lysate was determined,

and equal amounts of protein from the various groups were subjected to SDS-polyacrylamide gel electrophoresis, followed by transfer to PVDF membranes which were then blocked with nonfat milk. Then, the membranes were incubated overnight at $4{ }^{\circ} \mathrm{C}$ with the appropriate primary antibodies. Thereafter, the membranes were incubated with HRP-linked secondary antibody for $1 \mathrm{~h}$ at room temperature. Gel imaging system was used for image analysis.

\section{Statistical methods}

Measurement data consistent with normal 
distribution are presented as mean \pm SD. Univariate multivariate mean analysis was used for comparison among multiple groups, while SNK-q test was used for comparison between two groups. All statistical analyses were done with SPSS22.0 software package. Values of $p<$ 0.05 were considered indicative of statistically significant differences.

\section{RESULTS}

\section{Serum sex hormone levels}

In model mice, T, LH, FSH and leptin levels were markedly higher than in control mice. In contrast, compared with the model group, the levels of $\mathrm{T}$, $\mathrm{LH}, \mathrm{FSH}$ and leptin in resveratrol group were markedly decreased $(p<0.05)$. The results are shown in Table 2.

\section{Changes in body weight, ovarian weight and uterine weight of mice}

Compared with the control group, the body weight and ovarian weight of mice in the model group were significantly increased, while the uterine weight was markedly reduced $(p<0.05)$. However, compared with the model group, the body weight and ovarian weight of mice in the resveratrol group were significantly reduced, while the uterine weight was markedly increased $(p<0.05)$. These results are shown in Table 3 .

Table 2: Serum sex hormone levels of the mice

\section{Changes in mouse ovarian tissue}

Ovarian tissue of control mice was normal, the number of layers of follicular membrane was few and the number of corpus luteum was high. In the model group, the ovarian tissue structure was disordered, the follicular membrane layer was thickened, and there was evidence of follicular cystic dilatation. In contrast, ovarian tissue of mice in the resveratrol group was significantly improved, relative to that in the model group. The results are shown in Figure 1.

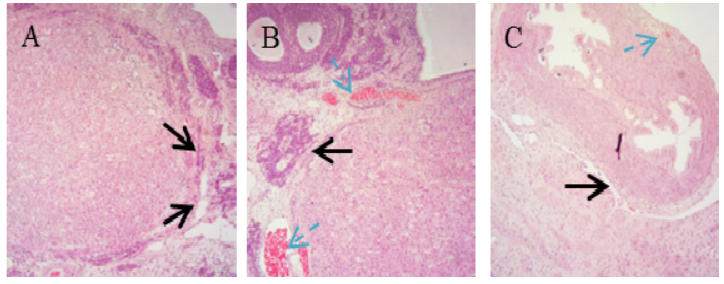

Figure 1: Changes in follicular tissues in the three groups. A: control group; F: model group; C: resveratrol group. Note: black arrows: the membrane of follicle; blue arrows: corpus luteum

\section{Apoptosis of ovarian cells}

There was higher degree of apoptosis of ovarian cells in model mice than in control. However, apoptosis of ovarian cells in the resveratrol group was significantly reduced, relative to model ( $p<$ 0.05). These results are presented in Figure 2.

\begin{tabular}{lcccc}
\hline Group & $\mathbf{T}(\mathbf{p g} / \mathbf{m L})$ & $\mathbf{L H}(\mathbf{p g} / \mathbf{m L})$ & FSH $(\mathbf{n g} / \mathbf{m L})$ & Leptin $(\mathbf{n g} / \mathbf{m L})$ \\
\hline Control & $1293.73 \pm 176.68$ & $2509.37 \pm 403.55$ & $14.23 \pm 2.46$ & $0.88 \pm 0.10$ \\
Model & $2757.77 \pm 441.09^{\mathrm{a}}$ & $8320.88 \pm 1187.75^{\mathrm{a}}$ & $47.65 \pm 7.20^{\mathrm{a}}$ & $2.64 \pm 0.36^{\mathrm{a}}$ \\
Resveratrol & $1683.11 \pm 235.36^{\mathrm{ab}}$ & $4433.43 \pm 733.14^{\mathrm{ab}}$ & $23.53 \pm 3.97^{\mathrm{ab}}$ & $1.25 \pm 0.16^{\mathrm{ab}}$ \\
$F$ & 368.10 & 747.31 & 727.13 & 938.24 \\
$P$-value & $<0.001$ & $<0.001$ & $<0.001$ & $<0.001$ \\
\hline
\end{tabular}

${ }^{a} P<0.05$, vs control; ${ }^{b} p<0.05$, vs model

Table 3: Body weight, ovarian weight and uterine weight of mice

\begin{tabular}{lccc}
\hline Group & Body weight of mice $\mathbf{( g )}$ & Ovarian weight $\mathbf{( m g )}$ & Uterine weight $(\mathbf{m g})$ \\
\hline Control & $24.11 \pm 2.21$ & $214.71 \pm 6.64$ & $315.11 \pm 11.05$ \\
Model & $33.12 \pm 3.63^{\mathrm{a}}$ & $337.45 \pm 17.42^{\mathrm{a}}$ & $233.90 \pm 6.33^{\mathrm{a}}$ \\
Resveratrol & $28.21 \pm 2.64^{\mathrm{ab}}$ & $281.71 \pm 5.68^{\mathrm{ab}}$ & $274.31 \pm 8.15^{\mathrm{ab}}$ \\
$F$ & 73.17 & 895.77 & 649.14 \\
$P$-value & $<0.001$ & $<0.001$ & $<0.001$ \\
\hline
\end{tabular}

${ }^{a} P<0.05$, vs control; ${ }^{b} p<0.05$, vs model

\section{Abnormal mitochondria and mitochondrial membrane potential of oocytes}

There were higher levels of abnormal mitochondria, while the mitochondrial membrane potential was significantly decreased in the model group, relative to control ( $p<0.05$; Table $4)$. However, compared with the model group, the number of abnormal mitochondria in the resveratrol group was markedly reduced ( $p<$ 0.05). Although the mitochondrial membrane potential was increased, no statistically significant difference was observed between resveratrol and the control groups.

\section{Levels of ROS in oocytes of mice}

Figure 3 and Table 4 show that ROS levels in 
model mice were significantly raised, relative to control. In contrast, ROS levels in mice in the resveratrol group were markedly decreased, relative to model mice $(p<0.05)$.

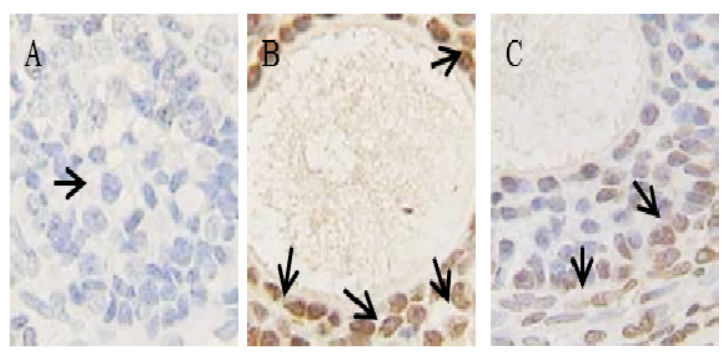

Figure 2: Apoptosis of ovarian cells in each group of mice. A: Control, B: model, C: resveratrol

Table 4: Abnormal mitochondria and mitochondrial membrane potential of oocytes in each group of mice

\begin{tabular}{lcc}
\hline Group & $\begin{array}{c}\text { Abnormal } \\
\text { mitochondrial } \\
\text { rate (\%) }\end{array}$ & $\begin{array}{c}\text { Mitochondrial } \\
\text { membrane } \\
\text { potential }\end{array}$ \\
\hline Control & $19.90 \pm 0.03$ & $1.13 \pm 0.05$ \\
Model & $54.65 \pm 0.10^{\mathrm{a}}$ & $0.99 \pm 0.12^{\mathrm{a}}$ \\
Resveratrol & $35.20 \pm 0.05^{\mathrm{ab}}$ & $1.03 \pm 0.07$ \\
$F$ & 49.85 & 21.47 \\
& $<0.001$ & $\mathrm{C}$
\end{tabular}

Figure 3: ROS in oocytes in the 3 groups. A: control group; B: model; C: resveratrol group

Table 4: ROS levels in oocytes of mice $(n=10)$

\begin{tabular}{ll}
\hline Group & ROS \\
\hline Control & $1.01 \pm 0.05$ \\
Model & $3.53 \pm 0.14^{\mathrm{a}}$ \\
Resveratrol & $1.51 \pm 0.06^{\mathrm{ab}}$ \\
$F$ & 6233.93 \\
$P$-value & $<0.001$ \\
\hline${ }^{a} P<0.05$, vs control, ${ }^{b} p<0.05$, vs PCOS mice
\end{tabular}

\section{Expression levels of apoptosis-related factors in oocytes of mice}

As shown in Table 5 , the expression levels of Bax, Bcl-2 and caspase- 9 in PCOS mice were markedly upregulated, relative to control. However, the expression levels of these apoptosis-related factors in the resveratrol group were markedly decreased, relative to PCOS mice $(p<0.05)$.
Table 5: Expression levels of apoptosis-related factors Bax, Bcl-2 and Caspase- 9 in oocytes of mice $(n=30)$

\begin{tabular}{lccc}
\hline Group & Bax & Bcl-2 & Caspase-9 \\
\hline Control & $1.02 \pm 0.04$ & $1.01 \pm 0.02$ & $0.98 \pm 0.05$ \\
Model & $1.62 \pm 0.06^{\mathrm{a}}$ & $1.21 \pm 0.04^{\mathrm{a}}$ & $1.30 \pm 0.04^{\mathrm{a}}$ \\
Resveratrol & $1.29 \pm 0.10^{\mathrm{ab}}$ & $1.09 \pm 0.05^{\mathrm{ab}}$ & $1.15 \pm 0.02^{\mathrm{ab}}$ \\
$F$ & 534.67 & 202.67 & 512.67 \\
$P$ & $<0.001$ & $<0.001$ & $<0.001$ \\
\hline${ }^{\mathrm{a} P}<0.05$, vs control, ${ }^{b} p<0.05 ;$ vs PCOS mice
\end{tabular}

\section{Levels of GPx and SOD in oocytes of mice}

There were significantly higher activities of GPx and SOD in PCOS mice than in control mice. However, the activities of GPx and SOD in the resveratrol group were markedly decreased, relative to model $(p<0.05$; Table 6$)$.

Table 6: Levels of GPx and SOD in oocytes of mice ( $\mathrm{n}$ $=30$ )

\begin{tabular}{lcc}
\hline Group & GPx & SOD \\
\hline Control & $1.00 \pm 0.07$ & $1.03 \pm 0.02$ \\
Model & $4.05 \pm 0.14^{\mathrm{a}}$ & $4.65 \pm 0.24^{\mathrm{a}}$ \\
Resveratrol & $3.16 \pm 0.29^{\mathrm{ab}}$ & $1.84 \pm 0.11^{\mathrm{ab}}$ \\
$F$ & 2038.70 & 4634.08 \\
$P$ & $<0.001$ & $<0.001$ \\
\hline${ }^{a} P<0.05$, vs control, ${ }^{b} p<0.05 ;$ vs PCOS model
\end{tabular}

\section{DISCUSSION}

Polycystic ovary syndrome (PCOS) is a disease characterized by persistent anovulation, androgen hyperactivity, and insulin resistance. At present, clinal treatment of PCOS with western medicine uses methods such as androgen reduction, ovulation promotion, and improvement of insulin resistance. Metformin is a frequently used drug in clinical practice, but the serious adverse effects associated with this drug limit its use. In recent years, the advantages of TCM in the treatment of PCOS have been gradually highlighted. In ancient Chinese medicine, there was no name for polycystic ovary syndrome. Based on its clinical symptoms and signs, it was mostly classified as "infertility", "metrorrhagia and metrostaxis" or "abdominal mass". It was believed that the disease was caused by the formation of sputum from accumulation of body fluids and water [7].

Resveratrol is a small molecule. Studies have found that it protects against development of a variety of diseases, and it is associated with high safety and good tolerance [8]. In this study, the effects of resveratrol on the treatment of polycystic ovary syndrome and oocytes were investigated.

In this study, a mouse model of DHEA-induced 
PCOS was established, and changes in serum sex hormones, body weight, ovarian weight, uterine weight and ovarian tissue were determined. It was found that DHEA effectively induced PCOS in a mouse model, and that resveratrol mitigated the $\mathrm{PCOS}$-induced changes in ovarian morphology in mice, and improved their body weights, ovarian weights and uterine weights. This may be related to the capacity of resveratrol to improve oocyte development in PCOS [9].

Studies in China and elsewhere have shown that hyperinsulinemia and insulin resistance are closely related to the occurrence of PCOS. In addition, a large number of peptide growth factors form a complex endocrine regulatory network, thereby promoting the occurrence of PCOS $[10,11]$. The results of this study showed that resveratrol significantly regulated the levels of serum sex hormones in PCOS mice. This may be because when PCOS occurs, a large amount of $\mathrm{LH}$ is secreted, and the persistent high level of LH stimulates the follicular membrane cells to produce excess androgens, thereby inhibiting the maturation of follicles into dominant follicles in the ovaries, resulting in a vicious cycle of excess androgens and persistent anovulation [12].

Resveratrol regulates homeostasis in the hypothalamic-pituitary-ovary axis by reducing the levels of $\mathrm{T}$ and $\mathrm{LH}$, thereby enhancing ovulation. Leptin, a protein expressed mainly in adipose tissue, regulates adipose tissue metabolism and reproductive functions. Some scholars have reported a close association between regulation of reproduction and PCOS, and suggested that PCOS may block the development of dominant follicles by inhibiting the effect of insulin-like growth factor-1 (IGF-1) on ovarian granulosa cells and follicular membrane cells [13].

The development and maturation of mammalian oocytes is a long and complex process. It may be regulated by a variety of mechanisms, resulting in changes in oocyte morphology and physiology [14]. It has been observed that oocyte quality in PCOS patients is markedly reduced, relative to healthy subjects. Mitochondria, as the power house of the cell, play critical roles in signal transduction, steroid hormone synthesis, granulocyte development and apoptosis [15]. The results of this study showed that PCOS induced obvious mitochondrial damage and oxidative stress in the ovarian tissue of mice in the model group. High concentrations of ROS damage mitochondria. In turn, the mitochondrial damage promotes production of ROS and causes oxidative stress, resulting in a vicious cycle which triggers apoptosis of oocytes [16].
The results obtained in this study indicate that resveratrol inhibited PCOS-induced oocyte apoptosis, mitigated oxidative stress and reduced mitochondrial damage.

\section{CONCLUSION}

Resveratrol significantly improves endocrine function and mitigates oxidative stress response in PCOS mice. Thus, resveratrol may be useful in the treatment of PCOS. However, there is need for further clinical trials.

\section{DECLARATIONS}

\section{Conflict of interest}

No conflict of interest is associated with this work.

\section{Contribution of authors}

We declare that this work was performed by the authors named in this article and all liabilities pertaining to claims relating to the content of this article will be borne by the authors. Zhifang Wang designed the study, supervised the data collection, and analyzed the data. Weiyan huang interpreted the data and prepared the manuscript for publication. Lili Duan, Meiyu Wu and Lei Wang supervised the data collection, analyzed the data and reviewed a draft of the manuscript.

\section{Open Access}

This is an Open Access article that uses a funding model which does not charge readers or their institutions for access and distributed under the terms of the Creative Commons Attribution License (http://creativecommons.org/licenses/by/ 4.0) and the Budapest Open Access Initiative (http://www.budapestopenaccessinitiative.org/rea d), which permit unrestricted use, distribution, and reproduction in any medium, provided the original work is properly credited.

\section{REFERENCES}

1. Indhavivadhana $S, \quad$ Rattanachaiyanont $M$, Wongwananuruk $T$, Techatraisak $K$, Rayasawath $N$, Dangrat $C$. Endometrial neoplasia in reproductive-aged Thai women with polycystic ovary syndrome. Int J Gynaecol Obstet 2018; 142(2): 170-175.

2. Albalawi FS, Daghestani MH, Daghestani $M H$, Eldali $A$, Warsy AS. rs4889 polymorphism in KISS1 gene, its effect on polycystic ovary syndrome development and anthropometric and hormonal parameters in Saudi women. J Biomed Sci 2018; 25(1): 50. 
3. Ge $Y$, Wang $K H$, Zha $Y T$, SHANG GL. Meta-Analysis on Adjuvant Therapy of Danzi Xiaoyao Pill for Polycystic Ovary Syndrome of Liver Depression Transforming into Fire Type. Chin Arch Tradit Chin Med 2018; 36(01): 6972.

4. Li G L, LiU J, Xie J. Effects of resveratrol on proliferation, apoptosis, migration and invasion of ovarian cancer SKOV-3 cells by regulating IL-6/JAK2/STAT3 signaling pathway. Acta Univ Med Anhui 2020; 55(5): 58-63.

5. Liu $B, X u Q$, Wang $D J$, Wang $Y$. Research Advances in the Function of Resveratrol on Hyperandrogen-Induced Abnormal Acetylation of Ovarian Cells. J Int Obstet Gynaecol 2019; 046(003): 342-345.

6. World Health Organization. Principles of laboratory animal care. WHO Chron 1985; 39: 51-56.

7. Endocrinology Group and Guideline Expert Group, Gynecology Branch, Chinese Medical Association. Chinese guidelines for diagnosis and treatment of polycystic ovary syndrome. Chin J Obstet Gynecol 2018; 53(1): 2-6.

8. Oh WY, Shahidi F. Antioxidant activity of resveratrol ester derivatives in food and biological model systems. Food Chem 2018; 261: 267-273.

9. Wang $Y$, Zhang M L, Li X. Progress of the effects of resveratrol on ovarian function and in vitro development of oocytes and embryos. Reprod Contracept 2019; 039(008): 664-667.

10. Li YF, Wan L, Ding Y. Effects of Heqi Powder on Endocrine Metabolism and Regulation of PIJK/AKT
Signaling Pathway in Polycystic Ovary Syndrome Model Rats. Chin J Chin Med 2019; 254(07): 125-128.

11. Wang $P$, Lin H, Huang Q. Clinical observation of Bushen Huoluo Prescription for treatment of kidney deficiency and blood stasis syndrome in adolescent polycystic ovary syndrome. Guiding J Tradit Chin Med Pharmacol 2018; 24(09): 63-65.

12. Daniel T, Peer B. Treatment of Paraphilic Disorders in Sexual Offenders or Men with a Risk of Sexual Offending with Luteinizing Hormone-Releasing Hormone Agonists: An Updated Systematic Review. J Sex Med 2019; 15(1): 77-93.

13. Szkudelska K, Deniziak M, Hertig I, Wojciechowicz $T$, Tyczewska M, Jaroszewska M, Szkudelski T. Effects of Resveratrol in Goto-Kakizaki Rat, a Model of Type 2 Diabetes. Nutrients 2019; 11(10): 2488.

14. Lee S, Jin JX, Taweechaipaisankul A, Kim GA, Ahn C, $L e e B C$. Sonic hedgehog signaling mediates resveratrol to improve maturation of pig oocytes in vitro and subsequent preimplantation embryo development. J Cell Physiol 2018; 233(6): 5023-5033.

15. Yan W, Qi XH. Resveratrol modulates SDF-1/CXCR4 signaling to inhibit hypoxia-ischemia-induced apoptosis of neurons from the brain of newborn rats. Chin $\mathrm{J}$ Pathophysiol 2018; 34(07): 33-38.

16. Folbergrova J, Jesina $P$, Kubova $H$, Otahal J. Effect of Resveratrol on Oxidative Stress and Mitochondrial Dysfunction in Immature Brain during Epileptogenesis. Mol Neurobiol 2018; 55(9): 7512-7522. 